
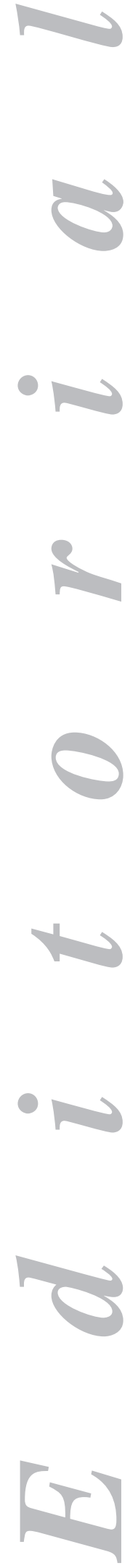

\section{Probiotics for Helicobacter pylori eradication therapy: Not ready for prime time}

Probiotics have been recently defined by the World Health Organization as "live microorganisms which, when administered in adequate amounts, may confer a health benefit" (1). Probiotics are a widely accepted therapy for both bacterial and viral acute gastroenteritis as well as diarrhea secondary to antibiotics, particularly in children. The most commonly used microorganisms for probiotic formulations in clinical practice include species of Lactobacillus, Bifidobacterium, Saccharomyces, Bacillus, and Streptococcus, as well as Escherichia coli (2). Multiple benefits of probiotics have been described against bacterial conditions, including the synthesis of substances with antimicrobial properties (lactic acid, hydrogen peroxide, bacteriocins), a competitive interaction with bacteria for adhesion receptors, and the modulation of the host's immune response (2). A field of gastroenterology where probiotics gained scientific interest in recent years is as adjuvants in the eradication therapy of Helicobacter pylori (H. pylori) infection.

In the present issue of Revista Española de Enfermedades Digestivas, Zheng et al. publish a meta-analysis assessing the impact of probiotic formulations with Lactobacillus for $H$. pylori eradication therapy (3). This is the eighth meta-analysis on the subject since 2007, and is surprising at first glance that it reports notably divergent results as compared to previous studies (4-10). As may be seen in table I, all meta-analyses reported to date, except two $(6,8)$, reveal a consistent, statistically significant increase in eradication rates and reduced side effects from antibiotic therapy. However, the meta-analysis by Zheng et al. (3) shows a notable increase in $H$. pylori eradication rates with no benefits on adverse effects. The authors state that these diverging results from the previously reported evidence are accounted for by their rigorous methodology, which includes: a) exclusion of non-randomized or incorrectly randomized

Table I. Reported meta-analyses that assessed the impact of various probiotic formulations on healing and side effect rates in connection with eradicating therapy for H. pylori infection

\begin{tabular}{lll}
\hline & \multicolumn{1}{c}{ Eradication rate } & \multicolumn{1}{c}{ Side effect rate } \\
\hline $\begin{array}{l}\text { Lactobacillus and } \\
\text { bifidobacterium }(4,5) \\
\text { Lactoferrin }(6,7)\end{array}$ & Statistically significant increase & Statistically significant reduction \\
& Statistically significant increase & $\begin{array}{l}\text { Statistically significant reduction } \\
\text { No benefit }\end{array}$ \\
$\begin{array}{l}\text { Fermented milk (8) } \\
\text { Lactobacillus (9) }\end{array}$ & Statistically significant increase & No benefit \\
$\begin{array}{l}\text { Saccharomyces } \\
\text { boulardii }(10)\end{array}$ & Statistically significant increase & Statistically significant reduction \\
\end{tabular}


studies; b) use of relative risks rather than odds ratios when estimating differences in adverse events between comparison groups; and c) exclusion of probiotic studies combining Lactobacillus and Saccharomyces boulardii, Bacillus subtilis, Bacillus clausi or Clostridium butyrium strains. Finally, the authors identified 9 randomized clinical trials that met their inclusion and exclusion criteria.

However, the fact that 5 of these 9 studies assessed probiotics combining different Lactobacillus strains and Bifidobacterium as well as Streptococcus strains, whereas only 4 used Lactobacillus-containing probiotics exclusively (3 different species though: L. gasseri, L. reuteri, L. casei), is strikingly outstanding (see table I in the original paper). The heterogeneity of meta-analyses having assessed the role of probiotics in the management of $H$. pylori infection, which jointly considered studies with different probiotic strains (sometimes different species of only one genus) at varying concentrations, is likely the primary reason precluding an adequate transfer or implementation of "statistical evidence" into "clinical practice". In this regard various studies were published last year that revealed no increase in $H$. pylori eradication rates or no reduced adverse effects following the use of probiotics in association with eradication therapy, both in adults and in children (11-14).

Most common causes of eradication therapy failure include antibiotic resistance and poor compliance (15). Recent studies in our setting have shown a higher effectiveness, approaching $90 \%$, of non-bismuth quadruple concomitant therapy $(16,17)$, hence this regimen is considered an effective alternative even in regions with high clarithromycin resistance rates. Adverse effects increase with antibiotic numbers and eradication therapy duration, which may directly influence compliance. It is in this field that probiotic implementation seems more rewarding, aiming at reducing adverse events and improving treatment compliance.

The results from this meta-analysis and the aforementioned studies (11-14) bolster our skepticism regarding their widespread use in eradication therapy for $H$. pylori infection. Figure 1 illustrates our personal view of the pros and cons the use of probiotics may presently have as an adjuvant to eradication therapy in routine clinical practice. On the other hand, we should importantly highlight that a vast majority of studies on the role of probiotics assessed their impact on the traditional triple therapy,

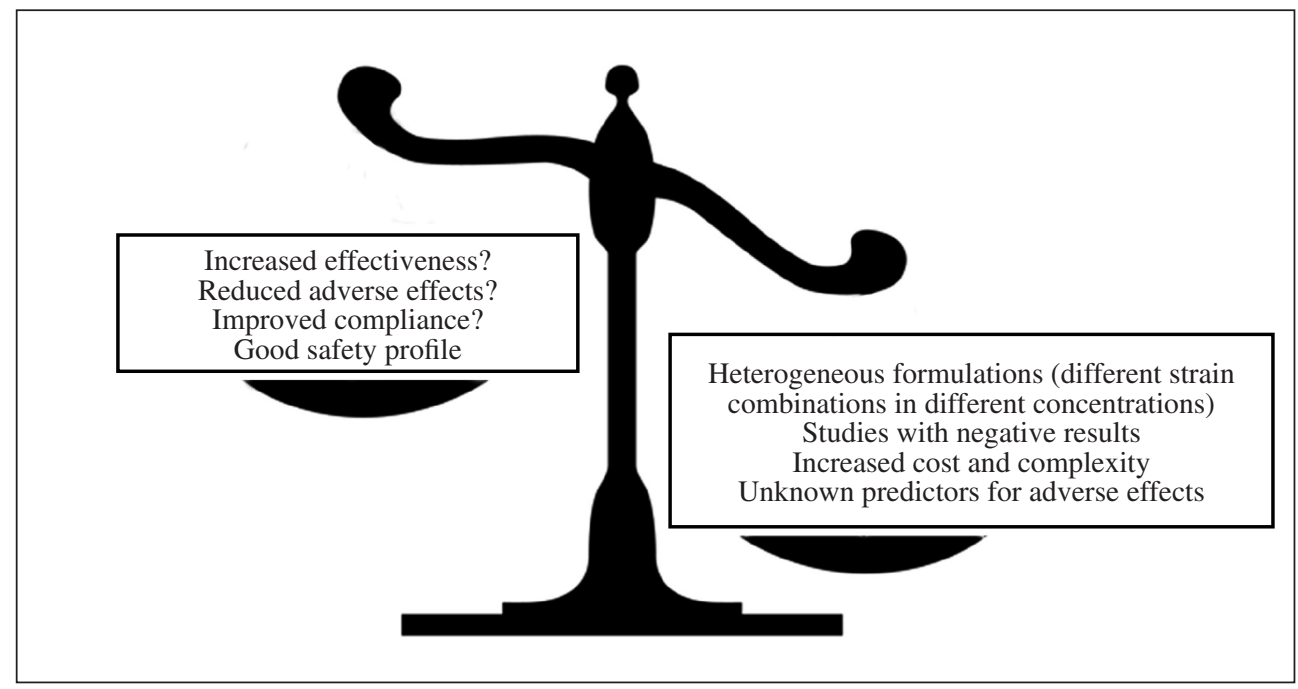

Fig. 1. Arguments for and against the use of probiotics as an adjuvant of $H$. pylori eradicating therapy in routine clinical practice. 
a regimen usually well tolerated. However, no studies so far have assessed the role of probiotics in the newer non-bismuth quadruple therapies, whether of "concomitant" or "sequential-concomitant hybrid" type. Also, no studies have been reported allowing to predict which patients have a higher risk for side effects, and could therefore be eligible for probiotic administration.

Lastly, we should take into account not only the statistical significance found for the studied combination (in our case the association between probiotic use and efficacy or adverse effects) but also the association's magnitude. Thus, as regards the magnitude of the potential increase in $H$. pylori eradication rates, this would be limited at most. The "number needed to treat" (NNT) estimated by Zheng et al. to heal one patient using Lactobacillus probiotics (as compared to standard eradication therapy) was 10, a considerably high figure. On the other hand, the relative risk estimated by these authors to reduce probiotic-related adverse events was 0.88 with a $95 \%$ confidence interval oscillating between 0.73 and 1.06 (that is, on the edge of statistical significance), and results were also considerably homogeneous (as measured by $\mathrm{I}^{2}=49 \%$ ).

To conclude, more robust and consistent evidence is apparently needed on the effectiveness of probiotics for $H$. pylori eradication therapy before they can be globally implemented in daily clinical practice. We should not forget that their prescription may complicate compliance and that their cost is substantial, as they are not presently supported by the National Health System. Similarly, we need to know their impact on the newer non-bismuth quadruple therapies, and to identify predictors for adverse events during eradication therapy in order to select the best candidates to benefit from their use. All these considerations are fully consistent with the opinion issued in the recently held "III Conferencia Española de Consenso" on H. pylori infection (18), which concludes that "a widespread use of probiotics in association with eradication therapy is not recommended", and that "currently, the most prudent option seems a recommendation to perform further studies in order to establish which strain, dose, and duration should probiotic therapy entail for the management of $H$. pylori infection, before their overall use in daily practice can be encouraged". To categorize evidence and recommendation strength the aforementioned consensus conference used the GRADE (Grading of Recommendations Assessment, Development and Evaluation Working Group) system (http://www.gradeworkinggroup.org/). This classification system considers the following factors to grade recommendation strength: a) balance between benefits and risks; b) evidence quality; c) values and preferences; and d) costs. In this way, their recommendation (against a widespread use of probiotics) was graded as "strong".

Javier Molina-Infante ${ }^{1}$ and Javier P. Gisbert ${ }^{2}$

${ }^{1}$ Department of Digestive Diseases. Hospital San Pedro de Alcántara. Cáceres, Spain.

${ }^{2}$ Department of Digestive Diseases. Hospital Universitario de La Princesa. Instituto de Investigación Sanitaria Princesa (IP) y Centro de Investigación Biomédica en Red de Enfermedades Hepáticas y Digestivas (CIBERehd). Madrid, Spain

\section{REFERENCES}

1. http://www.who.int/foodsafety/publications/fs_management/en/probiotics.pdf

2. Praitano MM, Iacono S, Francavilla R. Probiotics and Helicobacter pylori infection. Medicina Universitaria 2012;14:217-23. 
3. Zheng X, Lyu L, Mei Z. Lactobacillus-containing probiotic supplementation increases Helicoabcter pyloru eradication rate: evidence from a metanalysis. Rev Esp Enferm Dig 2013;105:445-53.

4. Tong JL, Ran ZH, Shen J, Zhang CX, Xiao SD. Meta-analysis: The effect of supplementation with probiotics on eradication rates and adverse events during Helicobacter pylori eradication therapy. Aliment Pharmacol Ther 2007;25:155-68.

5. Wang ZH, Gao QY, Fang JY. Meta-analysis of the efficacy and safety of Lactobacillus-containing and Bifidobacterium-containing probiotic compound preparation in Helicobacter pylori eradication therapy. J Clin Gastroenterol 2013;47:25-32.

6. Sachdeva A, Nagpal J. Meta-analysis: Efficacy of bovine lactoferrin in Helicobacter pylori eradication. Aliment Pharmacol Ther 2009;29:720-30.

7. Zou J, Dong J, Yu XF. Meta-analysis: The effect of supplementation with lactoferrin on eradication rates and adverse events during Helicobacter pylori eradication therapy. Helicobacter 2009;14:119-27.

8. Sachdeva A, Nagpal J. Effect of fermented milk-based probiotic preparations on Helicobacter pylori eradication: A systematic review and meta-analysis of randomized-controlled trials. Eur J Gastroenterol Hepatol 2009;21:45-53

9. Zou J, Dong J, Yu X. Meta-analysis: Lactobacillus containing quadruple therapy versus standard triple first-line therapy for Helicobacter pylori eradication. Helicobacter 2009;14:97-107.

10. Szajewska H, Horvath A, Piwowarczyk A. Meta-analysis: The effects of Saccharomyces boulardii supplementation on Helicobacter pylori eradication rates and side effects during treatment. Aliment Pharmacol Ther 2010;32:1069-79.

11. Medeiros JA, Goncalves TM, Boyanova L, Pereira MI, de Carvalho JN, Pereira AM, et al. Evaluation of helicobacter pylori eradication by triple therapy plus lactobacillus acidophilus compared to triple therapy alone. Eur J Clin Microbiol Infect Dis 2011;30:555-9.

12. Shavakhi A, Tabesh E, Yaghoutkar A, Hashemi H, Tabesh F, Khodadoostan M, et al. The effects of multistrain probiotic compound on bismuth-containing quadruple therapy for helicobacter pylori infection: A randomized placebo-controlled triple-blind study. Helicobacter 2013;18:280-4.

13. Padilla Ruiz M, Fernández Aguiar ME, Arce Nuñez M, Polo Amorín R. Lactobacillus rhamnosus GG supplementation to reduce side-effects of anti-Helicobacter pylori treatment. Rev Gastroenterol Peru 2013;33:121-30.

14. Du YQ, Su T, Fan JG, Lu YX, Zheng P, Li XH, et al. Adjuvant probiotics improve the eradication effect of triple therapy for Helicobacter pylori infection. World J Gastroenterol 2012;18:6302-7.

15. Graham DY, Lee YC, Wu MS. Rational Helicobacter pylori therapy: Evidence based medicine rather than medicine based evidence. Clin Gastroenterol Hepatol 2013 Jun 8.

16. Molina-Infante J, Romano M, Fernández-Bermejo M, Federico A, Gravina AG, Pozzati L, et al. Optimized non-bismuth quadruple therapies cure most patients with helicobacter pylori infection in populations with high rates of antibiotic resistance. Gastroenterology 2013;145:121-128.e1.

17. McNicholl AG, Marin AC, Molina-Infante J, Castro M, Barrio J, Ducons J, et al. Randomised clinical trial comparing sequential and concomitant therapies for Helicobacter pylori eradication in routine clinical practice. Gut 2013 May; en prensa.

18. Gisbert JP, Calvet X, Bermejo F, Boixeda D, Bory F, Bujanda L, et al. III Spanish Conference on Helicobacter pylori infection. Gastroenterol Hepatol 2013;36:340-74. 